\title{
Интенсиональная семантика логики классов Дж. Венна
}

\author{
Владимир Ильич Маркин \\ МГУ им. М.В. Ломоносова. \\ Российская Федерация, 119991, г. Москва, Ломоносовский пр-т, д. 27, корп. 4. \\ E-mail: markin@philos.msu.ru
}

\section{Мария Михайловна Легейдо}

МГУ им. М.В. Ломоносова.

Российская Федерация, 119991, г. Москва, Ломоносовский пр-т, д. 27, корп. 4.

E-mail: mlegeydo@yandex.ru

\begin{abstract}
Аннотация: В статье развивается предложенный В.И. Шалаком оригинальный подход к построению адекватных семантик для различных систем силлогистики. Его суть состоит в том, что субъектам и предикатам категорических высказываний сопоставляются в качестве значений формулы языка пропозициональной логики, а сами эти высказывания интерпретируются с использованием отношения логического следования. Семантика данного типа строится для логики классов Дж. Венна - силлогистики с нестандартным набором исходных простых высказываний: «Все $S$ есть все $P »(S a a P)$, «Все $S$ есть некоторые $P$ » $($ SaiP $)$, «Некоторые $S$ есть все $P$ » $($ SiaP $)$, «Некоторые $S$ есть некоторые $P$ » $(S i i P)$, «Ни один $S$ не есть $P »(S e P)$. Каждому из них соответствует одна из пяти круговых диаграмм Эйлера. Существуют две формализации силлогистики Венна с данным набором исходных констант: логика отношений между произвольными классами (система $\mathbf{C \Phi V ) ~ и ~ л о г и к а ~ о т н о ш е н и и ̆ ~ м е ж д у ~ н е п у с т ы м и ~ к л а с с а м и ~ ( с и с т е м а ~} \mathbf{C 4 V}$ ). Сначала семантика в стиле В.И. Шалака формулируется для системы $\mathbf{C \Phi V}$. Значениями общих терминов в ней являются любые пропозициональные формулы. SaaP значима, е.т.е. значения $S$ и $P$ следуют друг из друга; $S a i P$ значима, е.т.е. из значения $S$ следует значение $P$, но не наоборот; $\operatorname{SiaP}$ значима, е.т.е. из значения $P$ следует значение $S$, но не наоборот; $\mathrm{SeP}$ значима, е.т.е. из значения $S$ следует отрицание значения $P$; $\operatorname{SiiP}$ значима, е.т.е. значения $S$ и $P$ не следуют друг из друга и из первого не следует отрицание второго. В семантике для $\mathbf{C 4 V}$ значениями общих терминов являются только выполнимые пропозициональные формулы. Доказываются метатеоремы об адекватности данных семантик исчислениям $\mathbf{C \Phi V}$ и $\mathbf{C 4 V}$. Далее, семантика для $\mathbf{C \Phi V}$ модифицируется за счет замены классического следования на релевантное в определении значимости элементарных

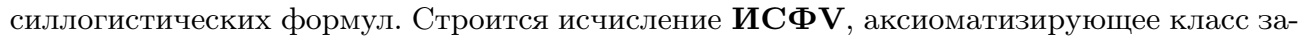
конов «релевантизированного» варианта силлогистики Венна. В заключение проводится сравнение всех предложенных систем по их дедуктивной силе.
\end{abstract}


Ключевые слова: силлогистика, Джон Венн, логика классов, интенсиональная семантика, формализация, аксиоматическое исчисление, логическое следование, релевантное следование

Для цитирования: Маркин В.И., Легейдо М.М. Интенсиональная семантика логики классов Дж. Венна // Логические исследования / Logical Investigations. 2019. Т. 25. № 2. C. 114-137. DOI: 10.21146/2074-1472-2019-25-2-114-137

\section{1. Интенсиональная интерпретация стандартных систем позитивной силлогистики}

В традиционной и современной логике доминирует сугубо экстенсиональное понимание силлогистики как логической теории, которая выделяет законы и способы корректных рассуждений, базирующиеся на отношениях между множествами индивидов. При такой трактовке эти множества выступают в качестве значений общих терминов (субъектов и предикатов категорических высказываний), а силлогистические константы рассматриваются как знаки различных отношений между двумя множествами (объемами понятий). Например, в фундаментальной силлогистике константа $a$ репрезентирует отношение теоретико-множественного включения объема субъекта в объем предиката, константа $i$ - отношение объемной совместимости субъекта и предиката (наличие общих элементов у соответствующих множеств) и т.д.

Чистый позитивный вариант ${ }^{1}$ фундаментальной силлогистики аксиоматизирует исчисление СФ [Бочаров, Маркин, 2010, с. 66-67], дедуктивно эквивалентное системе Дж. Шефердсона [Shepherdson, 1956]. Схемами аксиом СФ являются:

A0. Тавтологии классической логики высказываний;

A1. $(M a P \wedge S a M) \supset S a P$;

A2. $(M e P \wedge S a M) \supset S e P$;

A3. $S e P \supset P e S$;

A4. $S a S$;

A5. $S i P \supset S i S$;

A6. $S o P \supset S i S$;

A7. $S e P \equiv \neg S i P$;

A8. $S o P \equiv \neg S a P$.

Единственное правило вывода в $\mathbf{C \Phi}$ - modus ponens.

\footnotetext{
${ }^{1}$ Язык чистой позитивной силлогистики содержит только один тип нелогических символов - простые общие термины, в нем отсутствуют сингулярные термины и терминообразующие операторы.
} 
В том же самом языке сформулирован целый спектр логических систем, которые отличаются друг от друга классами выделяемых в них законов. Наиболее известной системой указанного типа является силлогистика Я. Лукасевича |Лукасевич, 1959], формализующая традиционную версию чистой позитивной силлогистики. С семантической точки зрения, силлогистические константы репрезентируют здесь те же самые отношения между множествами, что и в фундаментальной силлогистике, однако общим терминам в качестве значений сопоставляются не произвольные, а лишь непустые множества индивидов.

Исчисление, дедуктивно эквивалентное силлогистике Лукасевича, может быть получено из СФ добавлением новой схемы аксиом

A9. $S a P \supset$ SiP.

Следуя В.А. Смирнову, будем называть данное исчисление С4.

Менее известен в истории логики иной, альтернативный экстенсиональному подход к трактовке силлогистики. Г. Лейбниц в «Новых опытах о человеческом разумении» поставил задачу обоснования учения о силлогизме в интенсиональном ключе. Силлогистика, по его мнению, может рассматриваться как теория, основанная на отношениях между понятиями по содержанию, а не по объему. Тогда, с общими терминами связываются не множества индивидов, а содержания понятий, понимаемые как совокупности признаков индивидов. Из приводимых Лейбницем примеров видно, что в содержания понятий могут входить как положительные признаки (они указывают на наличие свойства у предметов), так и отрицательные признаки (фиксирующие отсутствие свойства у предметов).

При таком подходе силлогистические константы рассматриваются как знаки отношений между содержаниями двух понятий: одно из них связано с субъектом категорического высказывания, а другое с его предикатом. Поиску адекватной интерпретации силлогистических констант посвящена работа Лейбница «Элементы исчисления» Лейбниц, 1982, т. 3, с. 514-523].

Константа $а$ трактуется как знак отношения включения содержания предиката в содержание субъекта. Такая трактовка прямо вытекает из закона обратного отношения между содержаниями и объемами понятий. Таким образом, высказывание формы $S a P$ истинно, если и только если содержание его субъекта $S$ включает в себя все признаки из содержания предиката $P$. Именно в данном смысле общеутвердительное суждение есть мысль о том, что предикат содержится в субъекте.

Для частноутвердительных высказываний, содержащих силлогистическую константу $i$, Лейбниц предложил следующую интерпретацию: предикат в этом случае может, но не обязан содержаться в субъекте, достаточно, 
чтобы он содержался в каком-то виде субъекта, причем под видом имеется в виду понятие, более богатое, чем субъект, по содержанию. Здесь, правда, сразу возникает вопрос, а не приводит ли такая интерпретация высказываний вида $S i P$ к тому, что любое из них оказывается истинным. Действительно, если добавить к содержанию $S$ все признаки из содержания $P$, то мы как раз и получим искомый вид субъекта, в котором содержится предикат. Судя по всему, Лейбниц не считал, что любое объединение содержаний двух понятий дает содержание нового понятия. Если одно понятие содержит положительный признак «обладать (неким) свойством», а другое противоречащий ему отрицательный признак «не обладать (тем же самым) свойством», то в результате объединения содержаний получается противоречивое понятие. Поэтому, чтобы избежать ситуации, при которой все частноутвердительные суждения истинны, достаточно принять предпосылку о непротиворечивости понятий (об отсутствии в их содержаниях противоречащих друг другу признаков). Если исключить из сферы рассмотрения понятия с противоречивым содержанием, то лейбницевская трактовка константы $i$ эквивалентна следующей: высказывание формы SiP истинно, если и только если не существует противоречащих друг другу признаков, один из которых входит в содержание $S$, а другой в содержание $P$.

В.И. Маркин Маркин, 2001 построил формальную семантику для языка позитивной силлогистики, в которой общим терминам в качестве значений сопоставляются непустые и непротиворечивые (в указанном выше смысле) совокупности положительных и отрицательных признаков, а условия истинности атомарных формул $\operatorname{SaP}$ и $\operatorname{SiP}$ (а также противоречащих им формул $S o P$ и $S e P$ ) соответствуют лейбницевской трактовке категорических высказываний. Было установлено, что класс общезначимых в этой семантике формул совпадает с множеством теорем исчисления $\mathbf{C 4 .}$

В работе Маркин, 2002 был поставлен вопрос, как изменится множество законов силлогистики, если отказаться от предпосылки о непротиворечивости содержаний понятий, приписываемых в интенсиональной семантике общим терминам, сохранив при этом условия истинности формул: $S a P$ истинно, если и только если совокупность признаков, сопоставленная $P$, включается в совокупность признаков, сопоставленную $S$; $\mathrm{SiP}$ истинно, если и только если множества признаков, сопоставленные $S$ и $P$, не содержат противоречащих друг другу признаков. Оказалось, что не все теоремы фундаментальной силлогистики $\mathbf{C \Phi}$ (а тем более, не все теоремы C4) общезначимы в модифицированной указанным способом семантике. Класс общезначимых формул (при допущении в сферу рассмотрения понятий с противоречивыми содержаниями) аксиоматизирует исчисление 
ИСФ, которое получается из СФ за счет отбрасывания аксиомных схем $\mathbf{A 5}(\operatorname{SiP} \supset \operatorname{SiS})$ и $\mathbf{A} 6(S o P \supset S i S)$.

Оригинальный подход к построению семантик для систем позитивной силлогистики был сформулирован В.И. Шалаком [Шалак, 2015]. Он предложил сопоставлять в качестве значений общим терминам языка силлогистики формулы языка классической пропозициональной логики, а силлогистические константы интерпретировать с использованием отношения выводимости в классическом исчислении высказываний. По этой причине он назвал свою трактовку категорических высказываний синтаксической. Если значением термина $S$ является пропозициональная формула $\alpha$, а значением $P$ формула $\beta$, то $S a P$ означает, что из $\alpha$ в классической логике высказываний выводима $\beta$, а SiP означает, что из формул $\alpha$ и $\beta$ не выводимо противоречие (константа ложности).

Предложенную В.И. Шалаком семантику можно переформулировать без употребления константы ложности и с использованием вместо отношения классической выводимости его семантического аналога - отношения логического следования (в классической логике высказываний).

Пусть $\delta$ - функция, сопоставляющая каждому общему термину формулу языка классической логики высказываний, не содержащую иных связок кроме $\wedge, \vee$ и ᄀ. Для оценки силлогистических формул при той или иной интерпретации общих терминов задается двухместный метапредикат $\mathcal{F}$. Запись $\mathcal{F}(A, \delta)$ читается так: «силлогистическая формула $A$ значима при интерпретации общих терминов $\delta »$. Определение условий значимости формул силлогистического языка таково:

$$
\begin{array}{llrl}
\mathcal{F}(S a P, \delta) \Leftrightarrow \delta(S) \vDash \delta(P) ; & & \mathcal{F}(\neg A, \delta) \Leftrightarrow \dot{\mathcal{F}}(A, \delta) ; \\
\mathcal{F}(S e P, \delta) \Leftrightarrow \delta(S) \vDash \neg \delta(P) ; & & \mathcal{F}(A \wedge B, \delta) \Leftrightarrow \mathcal{F}(A, \delta) \dot{\wedge} \mathcal{F}(B, \delta) ; \\
\mathcal{F}(S i P, \delta) \Leftrightarrow \delta(S) \not \models \neg \delta(P) ; & & \mathcal{F}(A \vee B, \delta) \Leftrightarrow \mathcal{F}(A, \delta) \dot{\vee} \mathcal{F}(B, \delta) ; \\
\mathcal{F}(S o P, \delta) \Leftrightarrow \delta(S) \not \models \delta(P) ; & & \mathcal{F}(A \supset B, \delta) \Leftrightarrow \dot{\mathcal{F}}(A, \delta) \dot{\vee} \mathcal{F}(B, \delta) .
\end{array}
$$

Поясним это определение на примерах. Если термину $S$ функция $\delta$ приписывает формулу $q \wedge r$, а термину $P$ формулу $q \vee r$, то силлогистическая формула $S a P$ значима при этой интерпретации, так как из первой пропозициональной формулы логически следует вторая. Если же термину $S$ приписана $q \vee r$, а термину $P$ - формула $\neg q \wedge \neg r$, то силлогистическая формула $S i P$ не является значимой, поскольку из первой следует отрицание второй. Значимой при данной интерпретации оказывается противоречащая ей формула $S e P$.

Формула $A$ называется $\mathcal{F}$-общезначимой, е.т.е. $\mathcal{F}(A, \delta)$ при любой интерпретации общих терминов $\delta$. Множество $\mathcal{F}$-общезначимых формул, как 
показал В.И. Шалак Шалак, 2015], совпадает с множеством теорем силлогистического исчисления $\mathbf{C \Phi . ~}$

В русле развиваемого им подхода В.И. Шалак предложил также адекватную семантику для силлогистики Лукасевича (системы C4). Ее можно получить, если ограничить возможные значения общих терминов классически непротиворечивыми пропозициональными формулами. Иными словами, необходимо вместо функции $\delta$ (сопоставляющей общему термину любую пропозициональную формулу) ввести в семантику интерпретирующую функцию $\delta^{\prime}$, которая каждому общему термину приписывает в качестве значения некоторую выполнимую формулу. Новый предикат значимости $\mathcal{F}^{\prime}\left(A, \delta^{\prime}\right)$ определяется аналогично предикату $\mathcal{F}(A, \delta)$. Разница лишь в том, что функция $\delta$ в определении последнего меняется на $\delta^{\prime}$.

Формула $A$ называется $\mathcal{F}^{\prime}$-общезначимой, е.т.е. $\mathcal{F}^{\prime}\left(A, \delta^{\prime}\right)$ при любой интерпретации общих терминов $\delta^{\prime}$. Из полученного В.И. Шалаком результата Шалак, 2015] следует, что множество $\mathcal{F}^{\prime}$-общезначимых силлогистических формул равно множеству теорем системы $\mathbf{C 4}$.

«Синтаксическую» интерпретацию силлогистики, предложенную В.И. Шалаком, не следует противопоставлять интенсиональной интерпретации. Более того, можно считать предложенный им подход новой версией интенсиональной семантики для силлогистических систем. Дело в том, что нет никакой разницы в приписывании общим терминам пропозициональных формул $(q \vee r, q \wedge r, \neg q \wedge \neg r$ и т.п.) и в приписывании им бескванторных формул одноместной логики предикатов с единственной свободной переменной $x$ (например, $Q^{1}(x) \vee R^{1}(x), Q^{1}(x) \wedge R^{1}(x)$, $\left.\neg Q^{1}(x) \wedge \neg R^{1}(x)\right)$. С тем же успехом, в качестве значения общему термину можно сопоставлять первопорядковую формулу $A(x)$, которая содержит единственную переменную $x$ и либо является атомарной, либо представляет собой булеву комбинацию атомарных формул. Но, согласно Е.К. Войшвилло Войшвилло, 1967, предикат $A(x)$ как раз и фиксирует содержание понятия $x A(x)$. Следует, конечно, оговориться, что при таком подходе не будет охвачен весь класс понятий, но тем не менее мы будем иметь дело именно с понятиями и их содержаниями.

В рассмотренных ранее интенсиональных семантиках для систем C4 и ИСФ формула $S a P$ интерпретируется как выражающая утверждение о том, что содержание $P$ есть часть содержания $S$. Метаутверждение « $A(x) \vDash B(x) »$, согласно Войшвилло, также означает, что содержание понятия $x B(x)$ есть часть содержания понятия $x A(x)$. Значит, если субъекту $S$ сопоставлена формула $A(x)$, а предикату $P$ - формула $B(x)$, то «синтаксическая» интерпретация $S a P$ аналогична интенсиональной. 
Сходная аналогия между указанными интерпретациями имеет место и для других атомарных силлогистических формул. Так, $\mathrm{SeP}$ можно трактовать, с интенсиональной точки зрения, как утверждение о логической несовместимости содержаний субъекта и предиката. Но и при интерпретации В.И. Шалака условие значимости $S e P-\ll \delta(S) \vDash \neg \delta(P) »-$ фиксирует именно это отношение.

В.И. Маркин в Маркин, 2016а поставил вопрос о том, изменится ли класс общезначимых формул, если в условиях значимости заменить отношение классического следования на отношение релевантного следования.

Тогда семантика видоизменяется следующим образом. Вводится новый предикат значимости $\mathcal{I}$, который на атомарных силлогистических формулах определяется так:

$$
\begin{aligned}
& \mathcal{I}(S a P, \delta) \Leftrightarrow \delta(S) \vDash_{\text {rel }} \delta(P) ; \\
& \mathcal{I}(S e P, \delta) \Leftrightarrow \delta(S) \vDash_{\text {rel }} \neg \delta(P) ; \\
& \mathcal{I}(S i P, \delta) \Leftrightarrow \delta(S) \nvdash_{r e l} \neg \delta(P) ; \\
& \mathcal{I}(S o P, \delta) \Leftrightarrow \delta(S) \nvdash_{r e l} \delta(P),
\end{aligned}
$$

где $\delta$ - функция, сопоставляющая каждому общему термину формулу языка классической логики высказываний, не содержащую иных пропозициональных связок, кроме $\neg, \wedge$ и $\vee$, a $« \vDash_{r e l} »-$ следование в релевантной логике FDE. Условия значимости сложных формул остаются прежними.

Хорошо видно, что отличие $\mathcal{I}$ от ранее использовавшегося предиката значимости $\mathcal{F}$ состоит лишь в замене классического следования $(\vDash)$ на релевантное $\left(\vDash_{r e l}\right)$.

Силлогистическая формула $A$ называется $\mathcal{I}$-общезначимой, е.т.е. $\mathcal{I}(A, \delta)$ при любой интерпретации $\delta$.

Выяснилось, что множество $\mathcal{I}$-общезначимых формул не совпадает с множеством $\mathcal{F}$-общезначимых формул: первое строго включается во второе. Таким образом, при замене классического следования на релевантное в определении значимости силлогистических формул семантика перестает быть адекватной силлогистике СФ. Например, законы фундаментальной силлогистики $\operatorname{SiP} \supset \mathrm{SiS}$ и $\mathrm{SoP} \supset \mathrm{SiS}$ не являются $\mathcal{I}$-общезначимыми.

В работе Маркин, 2016а было доказано, что класс $\mathcal{I}$-общезначимых формул аксиоматизируется системой ИСФ.

Использование релевантного следования при интерпретации форм категорических высказываний представляет интерес именно в контексте построения интенсиональной семантики силлогистики. Е.К. Войшвилло неоднократно подчеркивал, что релевантное следование представляет собой отношение между высказываниями по информации, по их содержаниям, в то 
время как классическое следование фиксирует связь между высказываниям лишь по их значениям. С этой точки зрения, релевантное следование имеет интенсиональную природу, а классическое - экстенсиональную.

Адекватные семантики в духе В.И. Шалака с использованием как классического, так и релевантного следования можно сформулировать не только для упомянутых выше исчислений, но и для других известных систем позитивной силлогистики, которые формулируются в языке со стандартным набором силлогистических констант $\{a, e, i, o\}$ (см. [Маркин, 2016b]). Однако представляет интерес построение интенсиональных семантик для силлогистик с нестандартными силлогистическими константами. Одной из таких теорий является логика классов Дж. Венна.

\section{2. Логика классов Дж. Венна и ее формализация}

В первой главе своего фундаментального труда «Символическая логика» Venn, 1881 Дж. Венн дает достаточно подробное изложение оригинальной логической системы, которую можно охарактеризовать как теорию бинарных отношений между классами. Он выделяет пять базовых отношений между двумя множествами:

1. Равенство множеств;

2. Строгое включение первого множества во второе;

3. Строгое включение второго множества в первое;

4. Перекрещивание множеств;

5. Несовместимость (внеположенность) множеств.

Каждому из этих отношений соответствует одна из пяти диаграмм Эйлера-Жергонна, которые приведены на Рис. 1 .

Рис. 1. Диаграммы Эйлера-Жергонна
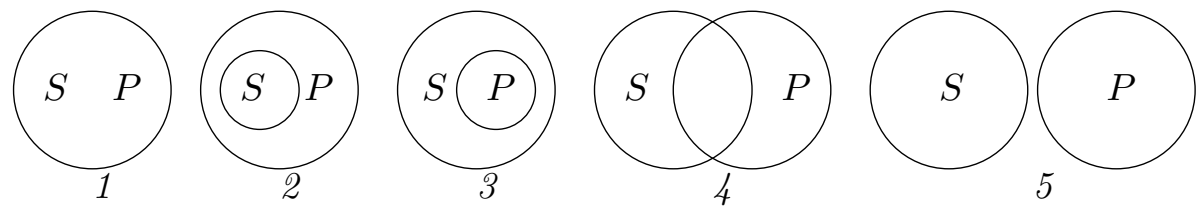

Венн ставит вопрос о способе выражения каждого из этих отношений в языке. При этом он существенно опирается на классификацию суждений, 
предложенную У. Гамильтоном, который считал необходимым при определении количественной характеристики категорического высказывания принимать во внимание квантификацию не только субъекта, но и предиката. Если в идущей от Аристотеля традиции категорические суждения делятся по количеству на общие и частные, то в учении Гамильтона они должны делиться на обще-общие, обще-частные, частно-общие и частно-частные.

Каждое из отношений 1-4 между двумя множествами адекватно выражается одной из четырех разновидностей утвердительных высказываний с квантифицированными субъектами и предикатами, отношение 5 обще-общим отрицательным высказыванием (которое Венн считает эквивалентным обычному общеотрицательному высказыванию):

1. Все $S$ есть все $P$;

2. Все $S$ есть некоторые $P$;

3. Некоторые $S$ есть все $P$;

4. Некоторые $S$ есть некоторые $P$;

5. Ни один $S$ не есть ни один $P$ (Ни один $S$ не есть $P$ ).

Далее Венн подробно разбирает силлогизмы первой фигуры, посылками которых являются высказывания этих пяти типов, выделяет среди них корректные и некорректные способы рассуждения.

Таким образом, логическая система Венна может рассматриваться как силлогистика с нестандартным набором силлогистических констант. Выбор именно этих констант обусловлен необходимостью репрезентации выделяемых базовых бинарных отношений между классами. Д.В. Дубаков и В.И. Маркин в Дубаков, Маркин, 2007] предложили следующую символическую запись венновских констант: для отношения 1 используется константа $a a$, для отношения 2 - константа $a i$, для отношения 3 константа $i a$, для отношения 4 - константа $i i$, для отношения 5 - стандартная константа $e$.

При принятии указанных обозначений атомарными формулами силлогистики Венна будут являться выражения видов SaaP, SaiP, SiaP, SiiP, $S e P$, где $S$ и $P$ - общие термины. Если формальную реконструкцию данной логической теории осуществлять в стиле Лукасевича, то есть с использованием в качестве основы классической логики высказываний, то в язык следует также ввести пропозициональные связки и скобки. Сложные формулы задаются при этом обычным способом. 
Существуют два аксиоматических исчисления, которые могут претендовать на роль современной реконструкции силлогистической логики классов Дж. Венна: система C4V Д.В. Дубакова и В.И. Маркина Дубаков, Маркин, 2007] и система $\mathbf{C \Phi V}$, построенная В.И. Маркиным в [Маркин,

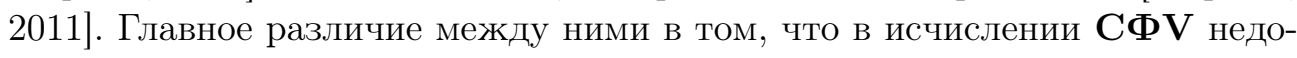
казуемы некоторые из так называемых «законов противоположностей», которые являются теоремами $\mathbf{C 4 V}$ :

$$
\neg(\text { SaaP } \wedge S e P), \neg(S a i P \wedge S e P), \neg(S i a P \wedge S e P) .
$$

Причина неоднозначности в выборе адекватной формализации силлогистики Венна, как отмечает В.И. Маркин Маркин, 2011, состоит в двойственности позиции самого автора по вопросу о принятии «законов противоположностей». С одной стороны, Венн четко заявляет, что каждому из пяти атомарных суждений соответствует ровна одна диаграмма, и тогда они должны быть попарно противоположными. С другой стороны, он дает такую алгебро-логическую интерпретацию этих суждений, при которой допускаются пустые классы в качестве значений субъектов и предикатов. Но при пустых $S$ и $P$ высказывания форм $S a a P$ и $S e P$ оказываются одновременно истинными; $S a i P$ и $S e P$ истинны при пустом $S$ и непустом $P$; а при пустом $P$ и непустом $S$ также будут вместе истинными высказывания форм $\operatorname{SiaP}$ и $\mathrm{SeP}$. Поэтому реконструкция силлогистики Венна может быть осуществлена в двух вариантах: «традиционном» (с исходной предпосылкой о непустоте терминов) и «фундаментальном» (без данной экзистенциальной предпосылки).

«Традиционный» вариант современной реконструкции силлогистики Венна (как логики бинарных отношений между непустыми классами) представляется исчислением $\mathbf{C 4} \mathbf{V}$, схемами аксиом которого наряду с пропозициональными тавтологиями (V0) являются:

V1. $(M a a P \wedge S a a M) \supset S a a P ; \quad$ V11. SeP $\supset P e S$;

V2. $(M a a P \wedge S a i M) \supset S a i P ; \quad$ V12. SaaS;

V3. $(M a i P \wedge S a a M) \supset S a i P ; \quad$ V13. $\neg($ SaaP $\wedge$ SaiP $)$;

V4. $(M a i P \wedge S a i M) \supset S a i P ; \quad$ V14. $\neg($ SaaP $\wedge$ SiaP $)$;

V5. $(M e P \wedge S a a M) \supset S e P ; \quad$ V15. $\neg(S a a P \wedge S i i P)$;

V6. $(M e P \wedge S a i M) \supset S e P ; \quad$ V16. $\neg($ SaiP $\wedge$ SiaP $)$;

V7. $S a a P \supset P a a S$;

V17. $\neg($ SaiP $\wedge$ SiiP $)$;

V8. SaiP $\supset$ PiaS;

V18. $\neg(S a a P \wedge S e P)$;

V9. SiaP $\supset$ PaiS;

V19. $\neg(S i i P \wedge S e P)$;

V10. SiiP $\supset$ PiiS; V20. $S a a P \vee S a i P \vee S i a P \vee S i i P \vee S e P$. 
Единственное правило вывода этого исчисления - modus ponens.

При построении «фундаментального» варианта реконструкции силлогистики Венна как логики любых (в том числе, и nустых) классов, аксиоматика видоизменяется. Постулатами соответствующей системы $\mathbf{C \Phi V}$ являются: правило modus ponens, V0, схемы аксиом V1-V17, V19-V20 системы $\mathbf{C 4 V}$, и две дополнительные схемы аксиом:

$$
\text { V21. } S e S \supset S e P ; \quad \text { V22. } S e S \supset(S a a P \vee S a i P) \text {. }
$$

Формулы последних двух типов доказуемы в $\mathbf{C 4 V}$, поэтому исчисление $\mathbf{C \Phi V}$ является подсистемой $\mathbf{C 4 V}$.

Д.В. Дубаков и В.И. Маркин показали [Дубаков, Маркин, 2007], что исчисление $\mathbf{C 4 V}$ рекурсивно эквивалентно системе позитивной фундаментальной силлогистики $\mathbf{C 4}$, строящейся в языке со стандартными константами, то есть эти исчисления погружаются друг в друга.

Существует перевод $v_{1}$, погружающий систему $\mathbf{C 4 V}$ в систему $\mathbf{C 4}$

$$
\begin{array}{ll}
v_{1}(\text { SaaP })=S a P \wedge P a S ; & v_{1}(\text { SiiP })=S i P \wedge S o P \wedge P o S ; \\
v_{1}(\text { SaiP })=S a P \wedge P o S ; & v_{1}(S e P)=S e P ; \\
v_{1}(\text { Sia } P)=S o P \wedge P a S ; & v_{1}(\neg A)=\neg v_{1}(A) ; \\
v_{1}(A \nabla B)=v_{1}(A) \nabla v_{1}(B), & \text { где } \nabla-\text { бинарная связка. }
\end{array}
$$

Существует также обратный перевод $v_{2}$, погружающий систему $\mathbf{C 4}$ в систему $\mathbf{C} 4 \mathbf{V}$ :

$$
\begin{array}{ll}
v_{2}(S a P)=S a a P \vee S a i P ; & v_{2}(S i P)=\neg S e P ; \\
v_{2}(S e P)=S e P ; & v_{2}(S o P)=\neg S a a P \wedge \neg S a i P ; \\
v_{2}(\neg A)=\neg v_{2}(A) ; & v_{2}(A \nabla B)=v_{2}(A) \nabla v_{2}(B) .
\end{array}
$$

В Маркин, 2011] отмечается, что перевод $v_{1}$ погружает также систему $\mathbf{C} \boldsymbol{\Phi} \mathbf{V}$ (формализующую «фундаментальный» вариант силлогистики Венна) в исчисление $\mathbf{C \Phi}$ (со стандартным набором силлогистических кон-

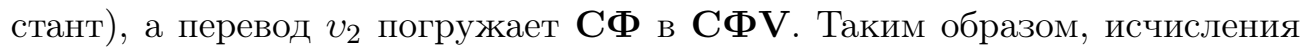
$\mathbf{C \Phi V}$ и $\mathbf{C \Phi}$ также являются рекурсивно эквивалентными.

\section{3. Интенсиональная семантика силлогистик $\mathrm{C} \Phi \mathrm{V}$ и $\mathrm{C4V}$}

Сформулируем сначала интенсиональную семантику, в которой высказывания языка силлогистики Венна интерпретируются через отношение классического следования между формулами пропозициональной логики, для системы $\mathbf{C \Phi V ~ - ~ ф о р м а л и з а ц и и ~ « ф у н д а м е н т а л ь н о и ̆ » ~ в е р с и и ~ с и л л о г и - ~}$ стики Венна. 
Пусть $\delta$ - функция, сопоставляющая каждому общему термину любую формулу языка классической логики высказываний, не содержащую иных пропозициональных связок, кроме $\neg, \wedge, \vee$. Зададим предикат $\mathcal{V}$ значимости

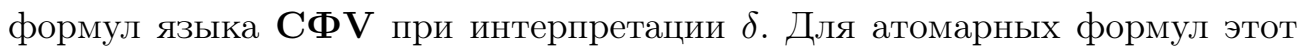
предикат определяется так:

$$
\begin{aligned}
& \mathcal{V}(\text { SaaP }, \delta) \Leftrightarrow \delta(S) \vDash \delta(P) \dot{\wedge} \delta(P) \vDash \delta(S) ; \\
& \mathcal{V}(\text { SaiP }, \delta) \Leftrightarrow \delta(S) \vDash \delta(P) \dot{\wedge} \delta(P) \not \models \delta(S) ; \\
& \mathcal{V}(\text { SiaP }, \delta) \Leftrightarrow \delta(S) \not \models \delta(P) \dot{\wedge} \delta(P) \vDash \delta(S) ; \\
& \mathcal{V}(\text { SiiP }, \delta) \Leftrightarrow \delta(S) \not \models \neg \delta(P) \dot{\wedge} \delta(S) \not \models \delta(P) \dot{\wedge} \delta(P) \not \models \delta(S) ; \\
& \mathcal{V}(\text { SeP }, \delta) \Leftrightarrow \delta(S) \vDash \neg \delta(P) .
\end{aligned}
$$

Для сложных формул условия значимости обычные.

Формула $A$ называется $\mathcal{V}$-общезначимой, если и только если $\dot{\forall} \delta \mathcal{V}(A, \delta)$, то есть $A$ значима при любой интерпретации $\delta$.

Условимся, что $\mathbf{L}$ - множество формул силлогистического языка со стандартными исходными константами $a, e, i, o$, a $\mathbf{L}_{\mathbf{V}}-$ множество формул языка силлогистики Венна, в котором исходными являются константы $a a, a i, i a, i i$ и $e$.

Для демонстрации адекватности данной семантики исчислению $\mathbf{C \Phi V}$ необходимо сначала доказать следующую метатеорему:

Теорема 1. Произвольная формула $A \in \mathbf{L}_{\mathbf{V}} \mathcal{V}$-общезначима, если и только если ее перевод $v_{1}(A) \mathcal{F}$-общезначим.

Доказательство. Обоснуем предварительно следующее утверждение:

$$
\dot{\forall} A \in \mathbf{L}_{\mathbf{V}} \dot{\forall} \delta\left(\mathcal{V}(A, \delta) \Leftrightarrow \mathcal{F}\left(v_{1}(A), \delta\right)\right) .
$$

Будем использовать возвратную индукцию по числу пропозициональных связок в формуле $A$.

I. $A=S a a P$.

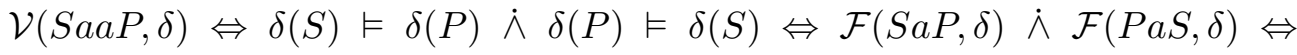
$\mathcal{F}(S a P \wedge P a S, \delta) \Leftrightarrow \mathcal{F}\left(v_{1}(S a a P), \delta\right)$.

II. $A=S a i P$.

$\mathcal{V}(S a i P, \delta) \Leftrightarrow \delta(S) \vDash \delta(P) \dot{\wedge} \delta(P) \not \models \delta(S) \Leftrightarrow \mathcal{F}(S a P, \delta) \dot{\wedge} \mathcal{F}(P o S, \delta) \Leftrightarrow$ $\mathcal{F}(S a P \wedge P o S, \delta) \Leftrightarrow \mathcal{F}\left(v_{1}(S a i P), \delta\right)$.

III. $A=\operatorname{SiaP}$.

$\mathcal{V}(S i a P, \delta) \Leftrightarrow \delta(S) \not \models \delta(P) \dot{\wedge} \delta(P) \vDash \delta(S) \Leftrightarrow \mathcal{F}(S o P, \delta) \dot{\wedge}(P a S, \delta) \Leftrightarrow$ $\mathcal{F}(S o P \wedge P a S, \delta) \Leftrightarrow \mathcal{F}\left(v_{1}(\right.$ SiaP $\left.), \delta\right)$. 
IV. $A=S i i P$.

$\mathcal{V}(\operatorname{SiiP}, \delta) \Leftrightarrow \delta(S) \quad \not \models \quad \neg \delta(P) \quad \dot{\wedge} \delta(S) \quad \not t \quad \delta(P) \quad \dot{\wedge} \delta(P) \quad \not \models \quad \delta(S) \quad \Leftrightarrow$ $\mathcal{F}(S i P, \delta) \dot{\wedge} \mathcal{F}(S o P, \delta) \wedge \mathcal{F}(P o S, \delta) \Leftrightarrow \mathcal{F}\left(v_{1}(S i i P), \delta\right)$.

V. $A=S e P$.

$\mathcal{V}(S e P, \delta) \Leftrightarrow \delta(S) \vDash \neg \delta(P) \Leftrightarrow \mathcal{F}(S e P, \delta) \Leftrightarrow \mathcal{F}\left(v_{1}(S e P), \delta\right)$.

VI. $A=\neg B$.

$\mathcal{V}(\neg B, \delta) \Leftrightarrow \dot{\neg} \mathcal{V}(B, \delta) \Leftrightarrow \dot{\neg} \mathcal{F}\left(v_{1}(B), \delta\right) \Leftrightarrow \mathcal{F}\left(\neg v_{1}(B), \delta\right) \Leftrightarrow \mathcal{F}\left(v_{1}(\neg B), \delta\right)$.

VII. $A=B \supset C$.

$\mathcal{V}(B \supset C, \delta) \Leftrightarrow \dot{\mathcal{V}}(B, \delta) \dot{\vee} \mathcal{V}(C, \delta) \Leftrightarrow \dot{\mathcal{F}}\left(v_{1}(B), \delta\right) \dot{\vee} \mathcal{F}\left(v_{1}(C), \delta\right) \Leftrightarrow$ $\mathcal{F}\left(v_{1}(B) \supset v_{1}(C), \delta\right) \Leftrightarrow \mathcal{F}\left(v_{1}(B \supset C), \delta\right)$.

Другие случаи, когда $A$ есть сложная формула, обосновываются аналогично.

Экививалентные преобразования в пяти базисных пунктах осуществляются на основе определений предикатов $\mathcal{V}$ и $\mathcal{F}$ и перевода $v_{1}$, в индуктивном переходе используется также индуктивное допущение о том, что утверждение леммы верно для формул с меньшим, чем у $A$, числом пропозициональных связок.

Из доказанного утверждения - $\dot{\forall} A \in \mathbf{L}_{\mathbf{V}} \dot{\forall} \delta\left(\mathcal{V}(A, \delta) \Leftrightarrow \mathcal{F}\left(v_{1}(A), \delta\right)\right)-$ по законам первопорядковой логики следует:

$$
\dot{\forall} A \in \mathbf{L}_{\mathbf{V}}\left(\dot{\forall} \delta \mathcal{V}(A, \delta) \Leftrightarrow \dot{\forall} \delta \mathcal{F}\left(v_{1}(A), \delta\right)\right) .
$$

Последнее означает, что произвольная формула $A \mathcal{V}$-общезначима в том и только в том случае, когда $\mathcal{F}$-общезначима формула $v_{1}(A)$.

Докажем теперь метатеорему об адекватности сформулированной выше семантики исчислению $\mathbf{C \Phi V . ~}$

Теорема 2. Произвольная формула $A \in \mathbf{L}_{\mathbf{V}}$ доказуема в исчислении $\mathbf{C \Phi V}$, если и только если $A-\mathcal{V}$-общезначимал формула.

Доказательство. В.И. Маркин Маркин, 2011] установил, что перевод $v_{1}$ погружает «фундаментальный» вариант силлогистики Венна $\mathbf{C \Phi V ~ в ~ с т а н - ~}$ дартную фундаментальную силлогистику СФ, то есть

$$
\dot{\forall} A \in \mathbf{L}_{\mathbf{V}}\left(\mathbf{C} \boldsymbol{\Phi} \mathbf{V} \vdash A \Leftrightarrow \mathbf{C} \boldsymbol{\Phi} \vdash v_{1}(A)\right) .
$$

Полученный В.И. Шалаком Шалак, 2015 результат свидетельствует о том, что все теоремы СФ, и только они, являются $\mathcal{F}$-общезначимыми формулами:

$$
\dot{\forall} B \in \mathbf{L}(\mathbf{C} \boldsymbol{\Phi} \vdash B \Leftrightarrow \dot{\forall} \delta \mathcal{F}(B, \delta)) .
$$


Данная равносильность справедлива и для $v_{1}$-переводов формул из $\mathbf{L}_{\mathbf{V}}$, поскольку эти переводы принадлежат $\mathbf{L}$ :

$$
\dot{\forall} A \in \mathbf{L}_{\mathbf{V}}\left(\mathbf{C} \boldsymbol{\Phi} \vdash v_{1}(A) \Leftrightarrow \dot{\forall} \delta \mathcal{F}\left(v_{1}(A), \delta\right)\right) .
$$

Согласно доказанной нами ранее Теореме 1 .

$$
\dot{\forall} A \in \mathbf{L}_{\mathbf{V}}\left(\dot{\forall} \delta \mathcal{V}(A, \delta) \Leftrightarrow \dot{\forall} \delta \mathcal{F}\left(v_{1}(A), \delta\right)\right) .
$$

Элементарным следствием утверждений (1), (3) и (4) является следующий тезис:

$$
\dot{\forall} A \in \mathbf{L}_{\mathbf{V}}(\mathbf{C} \boldsymbol{\Phi} \mathbf{V} \vdash A \Leftrightarrow \dot{\forall} \delta \mathcal{V}(A, \delta)) .
$$

Этот тезис означает, что множество теорем $\mathbf{C \Phi V}$ равно множеству $\mathcal{V}$-общезначимых формул.

Сформулируем далее адекватную семантику того же самого типа для системы $\mathbf{C 4 V}$ - формализации другого, «традиционного» варианта силлогистики Венна, то есть для его логики бинарных отношений между непустыми классами.

Вместо интерпретирующей функции $\delta$, сопоставляющей общим терминам любую формулу языка логики высказываний, не содержащую иных связок, кроме $\wedge, \vee$ и $\neg$, будем использовать функцию $\delta^{\prime}$, которая сопоставляет каждому общему термину некоторую выполнимую формулу указанного типа.

Предикат значимости силлогистической формулы $A$ при интерпретации $\delta^{\prime}-\mathcal{V}^{\prime}\left(A, \delta^{\prime}\right)$ - определяется так же, как и предикат $\mathcal{V}(A, \delta)$, с той лишь разницей, что вместо $\delta$ используется $\delta^{\prime}$. Формула $A$ называется $\mathcal{V}^{\prime}$-общезначимой, если и только если $\mathcal{V}^{\prime}\left(A, \delta^{\prime}\right)$ для любой интерпретации $\delta^{\prime}$.

Напомним, что предложенная В.И. Шалаком адекватная семантика системы $\mathbf{C} 4$ со стандартным набором исходных силлогистических констант может быть получена аналогичным образом: заменой в условиях значимости формул системы $\mathbf{C \Phi}$ интерпретирующей функции $\delta$ на функцию $\delta^{\prime}$. Действуя таким образом, вместо предиката значимости $\mathcal{F}(A, \delta)$ получим предикат $\mathcal{F}^{\prime}\left(A, \delta^{\prime}\right)$.

Теорема 3. Произвольная формула $A \in \mathbf{L}_{\mathbf{V}} \mathcal{V}^{\prime}$-общезначима, если и только если ее перевод $v_{1}(A) \mathcal{F}^{\prime}$-общезначим.

Доказателъство. Воспроизводим доказательство Теоремы 1 меняя $\mathcal{V}$ на $\mathcal{V}^{\prime}, \delta$ на $\delta^{\prime}, \mathcal{F}$ на $\mathcal{F}^{\prime}$.

Теорема 4. Произвольная формула $A \in \mathbf{L}_{\mathbf{V}}$ доказуема в исчислении $\mathbf{C 4 V}$, если и только если $A-\mathcal{V}^{\prime}$-общезначимая формула. 
Доказательство. Доказательство осуществляется по тому же плану, что и в Теореме 2. При этом используются: (1) результат Д.В. Дубакова и В.И. Маркина [Дубаков, Маркин, 2007] о том, что $v_{1}$ погружает систему C4V в C4; (2) результат В.И. Шалака ШШалак, 2015] о том, что множество теорем $\mathbf{C} 4$ равно множеству $\mathcal{V}^{\prime}$-общезначимых формул; (3) Теорема 3.

\section{4. Релевантизированная семантика языка силлогистики Венна. Система ИСФV}

В предыдущем разделе мы показали, что логические системы Венна, которые строились им как сугубо экстенсиональные логические теории, имеют адекватные интенсиональные интерпретации в духе В.И. Шалака. Можно сделать еще один шаг в «интенсионализации» семантики для силлогистики Венна: использовать в условиях значимости формул из $\mathbf{L}_{\mathbf{V}}$ вместо классического следования релевантное.

Пусть $\delta$ - функция, сопоставляющая каждому общему термину произвольную формулу языка классической логики высказываний, не содержащую иных пропозициональных связок, кроме $\neg, \wedge$ и $\vee, \mathrm{a} \ll \vDash_{r e l} 》-$ следование в релевантной логике FDE. Определим соответствующий предикат значимости $\mathcal{W}$ :

$$
\begin{aligned}
& \mathcal{W}(S a a P, \delta) \Leftrightarrow \delta(S) \vDash_{r e l} \delta(P) \dot{\wedge} \delta(P) \vDash_{r e l} \delta(S) ; \\
& \mathcal{W}(S a i P, \delta) \Leftrightarrow \delta(S) \vDash_{\text {rel }} \delta(P) \dot{\wedge} \delta(P) \nvdash_{\text {rel }} \delta(S) ; \\
& \mathcal{W}(S i a P, \delta) \Leftrightarrow \delta(S) \nvdash_{\text {rel }} \delta(P) \dot{\wedge} \delta(P) \vDash_{\text {rel }} \delta(S) \text {; } \\
& \mathcal{W}(S i i P, \delta) \Leftrightarrow \delta(S) \nvdash_{\text {rel }} \neg \delta(P) \dot{\wedge} \delta(S) \nvdash_{\text {rel }} \delta(P) \dot{\wedge} \delta(P) \nvdash_{\text {rel }} \delta(S) \text {; } \\
& \mathcal{W}(S e P, \delta) \Leftrightarrow \delta(S) \vDash_{\text {rel }} \neg \delta(P) \text {. }
\end{aligned}
$$

Для сложных формул условия значимости обычные.

Формула $A$ называется $\mathcal{W}$-общезначимой, если и только если $\dot{\forall} \delta \mathcal{W}(A, \delta)$.

Некоторые теоремы исчисления $\mathbf{C} \boldsymbol{\Phi \mathbf { V }}$ не являются $\mathcal{W}$-общезначимыми формулами. Адекватное «релевантизированной» семантике исчисление ИСФV получается из CФV отбрасыванием схем аксиом V21 и V22. Систему ИСФV можно также получить из «традиционной» версии силло-

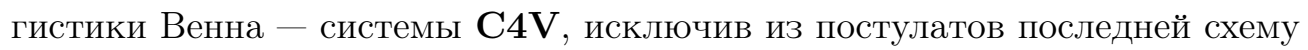
аксиом $\mathbf{V 1 8}(\neg(\operatorname{SaaP} \wedge \mathrm{SeP}))$.

Докажем, что множество теорем ИСФV совпадает с множеством $\mathcal{W}$-общезначимых формул.

Продемонстрируем сначала рекурсивную эквивалентность (взаимную

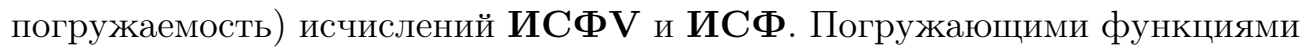
при этом будут заданные в предыдущем разделе переводы $v_{1}\left(\right.$ из $\mathbf{L}_{\mathbf{V}}$ в $\mathbf{L}$ ) и $v_{2}$ (из $\mathbf{L}$ в $\left.\mathbf{L} \mathbf{V}\right)$. 
Теорема 5. Перевод $v_{1}$ погружает систему ИСФV в систему ИСV, a перевод $v_{2}$ погружает систему ИСФ в систему ИСФV, т.е.

$\dot{\forall} A \in \mathbf{L}_{\mathbf{V}}\left(\mathbf{\Lambda C} \boldsymbol{\Phi} \mathbf{V} \vdash A \Leftrightarrow \mathbf{И C} \boldsymbol{\nabla} \vdash v_{1}(A)\right) u$

$\dot{\forall} A \in \mathbf{L}\left(\mathbf{\Lambda C} \boldsymbol{\Phi} \vdash A \Leftrightarrow \mathbf{\Lambda C} \boldsymbol{\Phi} \mathbf{V} \vdash v_{2}(A)\right)$.

Доказательство. Данная теорема будет доказываться с использованием следующего критерия взаимной погружаемости двух исчислений, в основе которого лежит известный критерий погружаемости В.А. Смирнова Смирнов, 2002, с. 127]:

«Исчисление $\mathbf{S}_{1}$ погружается в исчисление $\mathbf{S}_{\mathbf{2}}$ посредством фукнции $v_{1}$ (из множества формул $\mathbf{S}_{\mathbf{1}}$ в множество формул $\mathbf{S}_{\mathbf{2}}$ ), а исчисление $\mathbf{S}_{\mathbf{2}}$ погружается в исчисление $\mathbf{S}_{1}$ посредством фукнции $v_{2}$ (из множества формул $\mathbf{S}_{\mathbf{2}}$ в множество формул $\left.\mathbf{S}_{\mathbf{1}}\right)$, если и только если $(a)$ для каждой формулы $A$ языка $\mathbf{S}_{\mathbf{1}}$ имеет место: $\mathbf{S}_{\mathbf{1}} \vdash A \Rightarrow \mathbf{S}_{\mathbf{2}} \vdash v_{1}(A),(b)$ для каждой формулы $A$ языка $\mathbf{S}_{\mathbf{2}}$ имеет место: $\mathbf{S}_{\mathbf{2}} \vdash A \Rightarrow \mathbf{S}_{\mathbf{1}} \vdash v_{2}(A),(c)$ для каждой формулы $A$ языка $\mathbf{S}_{\mathbf{1}}$ имеет место: $\mathbf{S}_{\mathbf{1}} \vdash A \equiv v_{2}\left(v_{1}(A)\right),(d)$ для каждой формулы $A$ языка $\mathbf{S}_{\mathbf{2}}$ имеет место: $\mathbf{S}_{\mathbf{2}} \vdash A \equiv v_{1}\left(v_{2}(A)\right)$ ».

(a) Докажем, что $\dot{\forall} A \in \mathbf{L}_{\mathbf{V}}\left(\mathbf{U C} \boldsymbol{\Phi \mathbf { V }} \vdash A \Rightarrow \mathbf{И C} \boldsymbol{\Phi} \vdash v_{1}(A)\right)$.

Используем возвратную индукцию по длине доказательства формулы $A$ в исчислении $\mathbf{И C} \boldsymbol{\Phi V}$. Необходимо показать, что $v_{1}$-переводы всех аксиом ИCФV доказуемы в ИСФ, а также что правило modus ponens «сохраняет» доказуемость в ИСФ $v_{1}$-переводов формул из $\mathbf{L}_{\mathbf{V}}$.

V0. Переводы классических тавтологий также являются тавтологиями и поэтому доказуемы в ИСФ.

V1. $v_{1}((M a a P \wedge S a a M) \supset S a a P)=((M a P \wedge P a M) \wedge(S a M \wedge M a S)) \supset$ $(S a P \wedge P a S)$.

V2. $v_{1}((M a a P \wedge S a i M) \supset S a i P)=((M a P \wedge P a M) \wedge(S a M \wedge M o S)) \supset$ $(S a P \wedge P o S)$.

V3. $v_{1}((M a i P \wedge S a a M) \supset S a i P)=((M a P \wedge P o M) \wedge(S a M \wedge M a S)) \supset$ $(S a P \wedge P o S)$.

V4. $v_{1}((M a i P \wedge S a i M) \supset S a i P)=((M a P \wedge P o M) \wedge(S a M \wedge M o S)) \supset$ $(S a P \wedge P o S)$.

V5. $v_{1}((M e P \wedge S a a M) \supset S e P)=(M e P \wedge(S a M \wedge M a S)) \supset S e P$.

V6. $v_{1}((M e P \wedge S a i M) \supset S e P)=(M e P \wedge(S a M \wedge M o S)) \supset S e P$.

V7. $v_{1}(S a a P \supset P a a S)=(S a P \wedge P a S) \supset(P a S \wedge S a P)$.

V8. $v_{1}(S a i P \supset P i a S)=(S a P \wedge P o S) \supset(P o S \wedge S a P)$.

V9. $v_{1}(S i a P \supset P a i S)=(S o P \wedge P a S) \supset(P a S \wedge S o P)$.

V10. $v_{1}($ SiiP $\supset P i i S)=(S i P \wedge S o P \wedge P o S) \supset(P i S \wedge P o S \wedge S o P)$.

V11. $v_{1}(S e P \supset P e S)=S e P \supset P e S$.

V12. $v_{1}(S a a S)=S a S \wedge S a S$. 
V13. $v_{1}(\neg(S a a P \wedge S a i P))=\neg((S a P \wedge P a S) \wedge(S a P \wedge P o S))$.

V14. $v_{1}(\neg(S a a P \wedge S i a P))=\neg((S a P \wedge P a S) \wedge(S o P \wedge P a S))$.

V15. $v_{1}(\neg(S a a P \wedge S i i P))=\neg((S a P \wedge P a S) \wedge(S i P \wedge S o P \wedge P o S))$.

V16. $v_{1}(\neg($ Sai $P \wedge S i a P))=\neg((S a P \wedge P o S) \wedge(S o P \wedge P a S))$.

V17. $v_{1}(\neg(S a i P \wedge S i i P))=\neg((S a P \wedge P o S) \wedge(S i P \wedge S o P \wedge P o S))$.

V19. $v_{1}(\neg(S i i P \wedge S e P))=\neg((S i P \wedge S o P \wedge P o S) \wedge S e P)$.

V20. $v_{1}(S a a P \vee S a i P \vee S i a P \vee S i i P \vee S e P)=(S a P \wedge P a S) \vee(S a P \wedge P o S) \vee$ $(S o P \wedge P a S) \vee(S i P \wedge S o P \wedge P o S) \vee S e P$.

В работе [Дубаков, Маркин, 2007] приведены доказательства в исчислении $\mathbf{C} 4$ перечисленных $v_{1}$-переводов аксиом системы ИСФV, причем в этих доказательствах используются лишь те постулаты $\mathbf{C 4}$, которые принимаются в этом же качестве и в ее подсистеме ИСФ.

Modus ponens. Допустим, что $v_{1}(A \supset B)$ и $v_{1}(A)$ доказуемы в ИСФ. Поскольку $v_{1}(A \supset B)=v_{1}(A) \supset v_{1}(B)$, то $v_{1}(A) \supset v_{1}(B)$ является теоремой ИСФ. Но $v_{1}(A)$ тоже теорема этой системы. Следовательно, $v_{1}(B)$ доказуема в ИСФ.

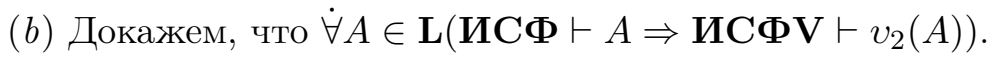

Используем тот же метод, что и в пункте $(a)$.

A0. Переводы классических тавтологий также являются тавтологиями и поэтому доказуемы в $\mathbf{U C \Phi V . ~}$

A1. $v_{2}((M a P \wedge S a M) \supset S a P)=((M a a P \vee M a i P) \wedge(S a a M \vee S a i M)) \supset$ $($ SaaP $\vee$ SaiP $)$.

Доказывается в ИСФV с использованием аксиом V1-V4.

A2. $v_{2}((M e P \wedge S a M) \supset S e P)=(M e P \wedge($ SaaM $\vee S a i M)) \supset S e P$. Доказывается в ИСФV с использованием аксиом V5-V6.

A3. $v_{2}(S e P \supset P e S)=S e P \supset P e S$.

Аксиома V11 системы ИСФV.

A4. $v_{2}(S a S)=S a a S \vee S a i S$.

Выводится из аксиомы V12 системы ИСФV.

A7. $v_{2}(S e P \equiv \neg S i P)=(S e P \equiv \neg \neg S e P)$;

A8. $v_{2}(S o P \equiv \neg S a P)=((\neg S a a P \wedge \neg S a i P) \equiv \neg($ SaaP $\vee S a i P))$.

Классические тавтологии.

Modus ponens. Обосновывается так же, как в предыдущем пункте.

(c) Докажем, что $\dot{\forall} A \in \mathbf{L}_{\mathbf{V}}\left(\mathbf{U C} \boldsymbol{\Phi} \mathbf{V} \vdash\left(A \equiv v_{2}\left(v_{1}(A)\right)\right)\right.$.

Доказательство ведется возвратной индукцией по числу пропозициональных связок в формуле $A$.

Пусть $A$ - атомарная формула одного из пяти типов. Если $A=S a a P$, то $v_{2}\left(v_{1}(A)\right)=v_{2}(S a P \wedge P a S)=(S a a P \vee S a i P) \wedge(P a a S \vee P a i S)$. Если $A=$ $S a i P$, то $v_{2}\left(v_{1}(A)\right)=v_{2}(S a P \wedge P o S)=(S a a P \vee S a i P) \wedge(\neg P a a S \wedge \neg P a i S)$. 
Если $A=S i a P$, то $v_{2}\left(v_{1}(A)\right)=v_{2}(S o P \wedge P a S)=(\neg S a a P \wedge \neg S a i P) \wedge$ $(P a a S \vee P a i S)$. Если $A=S i P$, то $\left.v_{2}\left(v_{1}(A)\right)=v_{2}(S i P \wedge S o P \wedge P o S)\right)=$ $\neg S e P \wedge(\neg S a a P \wedge \neg S a i P) \wedge(\neg P a a S \wedge \neg P a i S))$. Если $A=S e P$, то $v_{2}\left(v_{1}(A)\right)=$ $v_{2}(S e P)=S e P$.

Таким образом, необходимо доказать в ИCФV следующие теоремы: $S a a P \equiv((S a a P \vee S a i P) \wedge(P a a S \vee P a i S)) ;$

$S a i P \equiv((S a a P \vee S a i P) \wedge(\neg P a a S \wedge \neg P a i S)) ;$

$S i a P \equiv((\neg S a a P \wedge \neg S a i P) \wedge(P a a S \vee P a i S)) ;$

$S i i P \equiv(\neg S e P \wedge(\neg S a a P \wedge \neg S a i P) \wedge(\neg P a a S \wedge \neg P a i S)) ;$

$S e P \equiv S e P$.

В работе [Дубаков, Маркин, 2007] приведены доказательства указанных формул в исчислении $\mathbf{C 4 V}$, причем в доказательствах не используется схема аксиом V18, а значит эти формулы доказуемы и в системе $\mathbf{\Lambda C \Phi V . ~}$

В тех случаях, когда $A$ есть сложная формула, принимаем допущение, что для любой формулы $B$, содержащей меньше пропозициональных связок, чем $A$, верно, что $B \equiv v_{2}\left(v_{1}(B)\right)$ доказуема в системе $\mathbf{\Lambda C \Phi V}$.

Пусть $A=\neg B$. Согласно индуктивному допущению, $\mathbf{~} \mathbf{C \Phi \mathbf { V }} \vdash B \equiv$ $v_{2}\left(v_{1}(B)\right)$. По законам логики высказываний отсюда вытекает: ИC $\mathbf{\Phi V} \vdash$ $\neg B \equiv \neg v_{2}\left(v_{1}(B)\right)$. По определению переводов $v_{1}$ и $v_{2}$, имеем: $v_{2}\left(v_{1}(\neg B)\right)=$

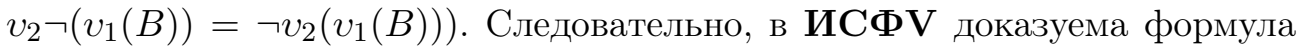
$\neg B \equiv v_{2}\left(v_{1}(\neg B)\right)$.

Пусть $A \mp B \supset C$. Согласно индуктивному допущению, ИС $\mathbf{\Phi} \vee \vdash B$ $v_{2}\left(v_{1}(B)\right)$ и $\mathbf{И C \boldsymbol { \Phi }} \vdash C \equiv v_{2}\left(v_{1}(C)\right)$. Отсюда по законам логики высказываний вытекает, что и формула $(B \supset C) \equiv\left(v_{2}\left(v_{1}(B)\right) \supset v_{2}\left(v_{1}(C)\right)\right)$ доказуема в этой системе. Но $v_{2}\left(v_{1}(B \supset C)\right)=v_{2}\left(v_{1}(B) \supset v_{1}(C)\right)=v_{2}\left(v_{1}(B)\right) \supset$ $v_{2}\left(v_{1}(C)\right)$. Следовательно, $\mathbf{И C} \boldsymbol{\Phi \mathbf { V }} \vdash(B \supset C) \equiv v_{2}\left(v_{1}(B \supset C)\right)$.

Остальные случаи, когда $A$ есть сложная формула, рассматриваются сходным образом.

$(d)$ Докажем, что $\dot{\forall} A \in \mathbf{L}\left(\mathbf{И C} \boldsymbol{\Phi} \vdash\left(A \equiv v_{1}\left(v_{2}(A)\right)\right)\right)$.

Снова используем возвратную индукцию по числу пропозициональных связок в формуле $A$.

Сначала рассмотрим четыре случая, когда $A$ - атомарная формула. Если $A=S a P$, то $v_{1}\left(v_{2}(A)\right)=v_{1}(S a a P \vee S a i P)=((S a P \wedge P a S) \vee(S a P \wedge$ $P o S))$. Если $A=S e P$, то $v_{1}\left(v_{2}(A)\right)=v_{1}(S e P)=S e P$. Если $A=S i P$, то $v_{1}\left(v_{2}(A)\right)=v_{1}(\neg S e P)=\neg v_{1}(S e P)=\neg S e P$. Если $A=S o P$, то $v_{1}\left(v_{2}(A)\right)=$ $v_{1}(\neg S a a P \wedge \neg S a i P)=(\neg(S a P \wedge P a S) \wedge \neg(S a P \wedge P o S))$.

Таким образом, необходимо доказать в ИСФ следующие теоремы: $S a P \equiv((S a P \wedge P a S) \vee(S a P \wedge P o S))$ $S e P \equiv S e P$ 
$S i P \equiv \neg S e P$

$S o P \equiv(\neg(S a P \wedge P a S) \wedge \neg(S a P \wedge P o S))$.

В работе [Дубаков, Маркин, 2007] приведены доказательства перечисленных формул с использованием схем $\mathbf{A 0}, \mathbf{A 7}, \mathbf{A 8}$ и правила modus ponens. Эти дедуктивные средства входят в число постулатов ИСФ, поэтому указанные формулы являются теоремами данной системы.

Индуктивный переход обосновывается аналогично пункту $(c)$.

Обосновав утверждения $(a)-(d)$, мы продемонстрировали взаимную погружаемость силлогистик ИСФV и ИСФ.

Теорема 6. Произвольная формула $A \in \mathbf{L}_{\mathbf{V}} \mathcal{W}$-общезначима, если и только если ее перевод $v_{1}(A) \mathcal{I}$-общезначим.

Доказательство. Доказательство данной метатеоремы аналогично доказательству Теоремы 1 .

Предварительно обосновывается следующее утверждение:

$$
\dot{\forall} A \dot{\forall} \delta\left(\mathcal{W}(A, \delta) \Leftrightarrow \mathcal{I}\left(v_{1}(A), \delta\right) .\right.
$$

Воспроизводим соответствующую часть доказательства Теоремы 1 , меняя предикат значимости $\mathcal{V}$ на $\mathcal{W}$, предикат значимости $\mathcal{F}$ на $\mathcal{I}$, классическое следование на релевантное.

Из данной леммы по законам первопорядковой логики получаем:

$$
\dot{\forall} A\left(\dot{\forall} \delta \mathcal{W}(A, \delta) \Leftrightarrow \dot{\forall} \delta \mathcal{I}\left(v_{1}(A), \delta\right)\right) .
$$

Последнее означает, что произвольная формула $A \in \mathbf{L}_{\mathbf{V}} \mathcal{W}$-общезначима в том и только в том случае, когда $\mathcal{I}$-общезначима $v_{1}(A)$.

Теорема 7. Для произвольной формулы $A \in \mathbf{L}_{\mathbf{V}}$ верно, что $v_{1}(A)$ доказуема в ИСФ, если и только если $v_{1}(A)-\mathcal{I}$-общезначимая формула.

Доказателъство. Семантические непротиворечивость и полнота силлогистики ИСФ относительно «релевантизированной» семантики доказаны В.И. Маркиным Маркин, 2016а. Произвольная формула из L доказуема в данной системе, если и только если она $\mathcal{I}$-общезначима. Поскольку перевод $v_{1}$ любой формулы «венновского» языка из $\mathbf{L}_{\mathbf{V}}$ принадлежит множеству формул стандартного языка $\mathbf{L}$, то указанная равносильность (доказуемости в ИСФ и $\mathcal{I}$-общезначимости) имеет место и для него.

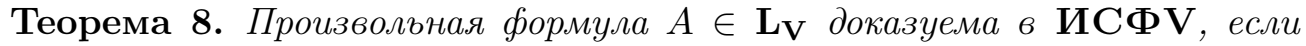
и только если $A \mathcal{W}$-общезначима. 
Доказательство. Согласно Теореме 5 , доказуемость произвольной

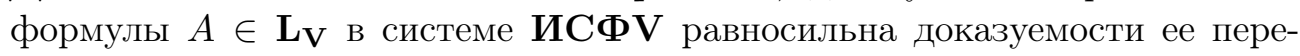
вода $v_{1}(A)$ в системе ИСФ. Согласно Теореме 7, доказуемость $v_{1}(A)$ в ИСФ равносильна $\mathcal{I}$-общезначимости $v_{1}(A)$. И, согласно Теореме 6 . $\mathcal{I}$-общезначимость $v_{1}(A)$ равносильна $\mathcal{W}$-общезначимости формулы $A$.

В заключение, сравним три рассмотренных нами системы силлогистики в языке с исходными константыми $a a, a i, i a, i i$ и $e$ по их дедуктивной силе.

Системы $\mathbf{C 4 V}, \mathbf{C \Phi V}$ и ИС $\mathbf{\Phi V}$ очень близки по классам тех силлогистических принципов, которые являются законами этих систем. В этом язык силлогистики Венна серьезно отличается от стандартного силлогистического языка, где переход от «традиционной» к «фундаментальной» версии силлогистики сопровождается отказом от многих известных законов: девяти модусов простого категорического силлогизма, закона обращения для высказываний типа $a$, многих умозаключений по логическому квадрату и др.

В каждом из исчислений $\mathbf{C 4 V}, \mathbf{C \Phi V}$ и $\mathbf{~} \mathbf{C \Phi V}$ доказуемы:

- 52 модуса категорического силлогизма (по 13 в каждой фигуре);

- одни и те же законы обращения;

- закон силлогистического тождества $S a a S$;

- закон «исключенного шестого» $S a a P \vee S a i P \vee S i a P \vee S i i P \vee S e P$;

- семь из десяти «законов противоположностей» вида $\neg(S y P \wedge S q P)$, где $y$ и $q$ - различные константы из множества $\{a a, a i, i a, i i, e\}$.

Имеются однако и различия между указанными системами.

Только в $\mathbf{C 4 V}$ доказуемы три «закона противоположностей»:

$$
\neg(S a a P \wedge S e P), \neg(S a i P \wedge S e P), \neg(S i a P \wedge S e P) .
$$

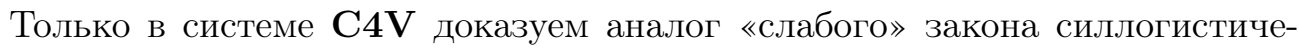
ского тождества:

$$
\neg S e S .
$$

В системе $\mathbf{C \Phi V ~ д о к а з у е м ы ~ о с л а б л е н и я ~ э т о г о ~ з а к о н а : ~}$

$$
S e S \supset S e P, \quad S e S \supset(S a a P \vee S a i P) .
$$

А в «релевантизированной» версии «фундаментального» варианта силлогистики Венна $\mathbf{И C \Phi V ~ н е ~ д о к а з у е м ы ~ и ~ о н и . ~}$ 


\section{Литература}

Бочаров, Маркин, 2010 - Бочаров В.А., Маркин В.И. Силлогистические теории. М.: Прогресс-Традиция, 2010. 333 с.

Войшвилло, 1967 - Войшвилло Е. К. Понятие. М.: Изд-во Моск. ун-та, 1967. 287 с.

Дубаков, Маркин, 2007 - Дубаков Д.В., Маркин В.И. Система силлогистики с исходными константами, соответствующими круговым диаграммам // Труды научно-исследовательского семинара Логического центра Института философии РАН. Вып. ХVIII. М.: ИФ РАН, 2007. С. 63-75.

Лейбниц, 1982 - Лейбнии Г.В. Сочинения в 4-х томах. М.: Мысль, 1982-1989.

Лукасевич, 1959 - Лукасевич Я. Аристотелевская силлогистика с точки зрения современной формальной логики. М.: Изд-во иностр. лит-ры, 1959. 313 с.

Маркин, 2001 - Маркин В.И. Интенсиональная семантика традиционной силлогистики // Логические исследования. Вып. 8. М.: Наука, 2001. С. 82-91.

Маркин, 2002 - Маркин В.И. Фундаментальная силлогистика с интенсиональной точки зрения // Логические исследования. Вып. 9. М.: Наука, 2002. С. 119 130.

Маркин, 2011 - Маркин В.И. Формальные реконструкции силлогистики Венна // Вестник Московского университета. Серия 7: Философия. 2011. № 1. C. $63-73$.

Маркин, 2016а - Маркин В.И. Интерпретация категорических высказываний в терминах релевантного следования // Логические исследования. 2016. Т. 22. № 1. C. $70-81$.

Маркин, 2016b - Маркин В.И. Семантика позитивных силлогистик и релевантное следование // Логико-философские штудии. 2016. Т. 13, № 2. С. 34-39.

Смирнов, 2002 - Смирнов В. А. Логические методы анализа научного знания. М.: Эдиториал УРСС, 2002. 263 с.

Шалак, 2015 - Шалак В.И. Синтаксическая интерпретация категорических атрибутивных высказываний // Логические исследования. 2015. Т. 21. № 1. C. $60-78$.

Shepherdson, 1956 - Shepherdson J.C. On the Interpretation of Aristotelian Syllogistic // Journal of Symbolic Logic. 1956. Vol. 21. No. 2. P. 137-147.

Venn, 1881 - Venn J. Symbolic Logic. London: Macmillan and Co., 1881. 446 p. 


\title{
Vladimir I. MARKin, MARia M. LEgeydo
}

\section{Intensional Semantics for J. Venn's Logic of Classes}

\author{
Vladimir I. Markin \\ Lomonosov Moscow State University, \\ 27/4 Lomonosovskiy prospect, Moscow, 119991, Russian Federation. \\ E-mail: markin@philos.msu.ru

\section{Maria M. Legeydo} \\ Lomonosov Moscow State University, \\ 27/4 Lomonosovskiy prospect, Moscow, 119991, Russian Federation. \\ E-mail: mlegeydo@yandex.ru
}

\begin{abstract}
In this article we expound V.I. Shalack's approach to the adequate semantics for different syllogistic systems construction. The point is that the formulas of propositional logic are associated with the subject and the predicate of the categorical propositions as their meanings. The propositions themselves are interpreted with the help of logical entailment. We constructed semantics of this type for the J. Venn's syllogistic with non-standard primitive propositions: "All $S$ is all $P$ " $($ SaaP $)$, "All $S$ is some $P$ " (SaiP), "Some $S$ is all $P$ " $($ SiaP $)$, "Some $S$ is some $P$ " $(S i i P)$, "No $S$ is $P$ " $(S e P)$. Each of them corresponds to one of the Euler diagrams. There are two kinds of Venn's syllogistic formalization: one is the theory of the relations between arbitrary classes and another is the theory of the relations between non-empty classes. We construct Shalack's type semantics for the first formalization. We introduce the function $\delta$ puts arbitrary propositional formulas in correspondence with the general terms. Let the general terms $S$ and $P$ be interpreted by propositional formulas $A$ and $B$. SaaP is true under this interpretation iff $A$ entails $B$ and $B$ entails $A ; S a i P$ is true iff $A$ entails $B$ and $B$ doesn't entail $A ; S i a P$ is true iff $A$ doesn't entail $B$ and $B$ entails $A ; S e P$ is true iff $A$ entails the negation of $B$; SiiP is true iff $A$ doesn't entail $B, B$ doesn't entail $A$ and $A$ doesn't entail the negation of $B$. Truth definitions for complex syllogistic formulas are standard. The adequate semantics for the second formalization of Venn's syllogistic is constructed by changing the interpretation of general terms: we assign to them only satisfiable propositional formulas. Soundness and completeness theorems are proved for both types of the syllogistic. Also we construct the semantics where we use relevant (FDE) entailment in favour of classical entailment in the truth definitions of Venn's syllogistic formulas. We formulate the syllogistic calculus which is adequate to this semantics. In conclusion we compare the deductive power of three Venn's type syllogistics.
\end{abstract}

Keywords: syllogistic, John Venn, logic of classes, intensional semantics, formalization, axiomatic calculus, logical entailment, relevant entailment

For citation: Markin V.I., Legeydo M.M. "Intensional'naya semantika logiki klassov Dzh. Venna" [Intensional Semantics for J. Venn's Logic of Classes], Logicheskie Issledovaniya / Logical Investigations, 2019, Vol. 25, No. 2, pp. 114-137. DOI: 10.21146/2074-1472-2019-252-114-137 (In Russian) 


\section{References}

Bocharov, Markin, 2010 - Bocharov, V.A., Markin, V.I. Sillogisticheskie teorii [Syllogistic theories]. Moscow: Progress-tradition Publ., 2010. 333 pp. (In Russian)

Dubakov, Markin, 2007 - Dubakov, D.V., Markin, V.I. "Sistema sillogistiki s ishodnymi konstantami, sootvetstvuyushimi krugovym diagrammam" [The syllogistic system with initial constants corresponding to circular diagrams], in: Trudy nauchno-issledovatelskogo seminara Logicheskogo centra Instituta filosofii RAN [Proceedings of scientific research seminar of Logical center Institute of Philosophy RAS ]. Vol. XVIII. Moscow: Institute of Philosophy RAS, 2007, pp. 63-75. (In Russian)

Leibniz, 1982 - Leibniz, G. Sochineniya $v$ 4-h tomah [Writings in 4 volumes]. Moscow: Mysl', 1982-1989. (In Russian)

Łukasiewicz, 1959 - Łukasiewicz, J. Aristotelevskaya sillogistika s tochki zreniya sovremennoj formal'noj logiki [Aristotile's sillogistic from the standpoint of modern formal logic]. Moscow: Foreign Literature Publ., 1959. 313 pp. (In Russian)

Markin, 2001 - Markin, V.I. "Intensional'naya semantika tradicionnoj sillogistiki" [Intensional semantics for traditional syllogistic], in: Logicheskie issledovaniya [Logical investigations]. Vol. 8. Moscow: Nauka, 2001, pp. 82-91. (In Russian)

Markin, 2002 - Markin, V.I. "Fundamental'naya sillogistika s intensional'noj tochki zreniya" [Fundamental syllogistic from the intensional standpoint], in: Logicheskie issledovaniya [Logical investigations]. Vol. 9. Moscow: Nauka, 2002, pp. 119-130. (In Russian)

Markin, 2011 - Markin, V.I. "Formal'nye rekonstrukcii sillogistiki Venna" [Formal reconstructions of Venn's syllogistic], Vestnik Moskovskogo universiteta. Seriya 7: Filosofiya [Moscow University Bulletin. Ser. 7: Philosophy], 2011, No. 1, pp. 6373. (In Russian)

Markin, 2016a - Markin, V.I. "Interpretaciya kategoricheskih vyskazyvanij v terminah relevantnogo sledovaniya" [Interpretation of categorical propositions in terms of relevant entailment], Logicheskie issledovaniya [Logical investigations], 2016, Vol. 22, No. 1, pp. 70-81. (In Russian)

Markin, 2016b - Markin, V.I. "Semantika pozitivnyh sillogistik i relevantnoe sledovanie" [Semantics of positive syllogistics and relevant entailment], Logikofilosofskie shtudii [Logical and philosophical studies], 2016, Vol. 13, No. 2, pp. 3439. (In Russian)

Shalack, 2015 - Shalack, V.I. "Sintaksicheskaya interpretaciya kategoricheskih atributivnyh vyskazyvanij" [Syntactic interpretation of categorical attributive propositions], Logicheskie issledovaniya [Logical investigations], 2015, Vol. 21, No. 1, pp. 60-78. (In Russian)

Shepherdson, 1956 - Shepherdson, J.C. "On the Interpretation of Aristotelian Syllogistic", Journal of Symbolic Logic, 1956, Vol. 21, No. 2, pp. 137-147.

Smirnov, 2002 - Smirnov, V.A. Logicheskie metody analiza nauchnogo znaniya [Logical methods of the analyses of scientific knowledge]. Moscow: Editorial URSS, 2002. 263 pp. (In Russian) 
Venn, 1881 - Venn, J. Symbolic Logic. London: Macmillan and Co., 1881. 446 pp.

Voishvillo, 1967 - Voishvillo, E.K. Ponyatie [Concept]. Moscow: Moscow St. Univ. Publ., 1967. 287 pp. (In Russian) 